\title{
Highly pathogenic adapted HIV-1 strains limit host immunity and dictate rapid disease progression
}

\author{
Judith Dalmau ${ }^{a}, *$, Margalida Rotger ${ }^{b, *}$, Itziar Erkizia ${ }^{a}$, Andri Rauch', \\ Pedro Reche ${ }^{\mathrm{d}}$, Maria Pino ${ }^{\mathrm{a}}$, Anna Esteve ${ }^{\mathrm{f}}$, Eduard Palou ${ }^{\mathrm{g}}$, \\ Christian Brander ${ }^{a, i}$, Roger Paredes ${ }^{a}$, Pham Phung ${ }^{\mathrm{e}}$, \\ Bonaventura Clotet $^{\mathrm{a}, \mathrm{h}}$, Amalio Telenti ${ }^{\mathrm{b}}$, Javier Martinez-Picado ${ }^{\mathrm{a}, \mathrm{h}, \mathrm{i}}$, \\ Julia G. Pradoa , The CoRP Study Group
}

\begin{abstract}
Objective: The study of HIV-1 rapid progressors has been limited to specific case reports. Nevertheless, identification and characterization of the viral and host factors involved in rapid progression are crucial when attempting to uncover the correlates of rapid disease outcome.
\end{abstract}

Design: We carried out comparative functional analyses in rapid progressors $(n=46)$ and standard progressors $(n=46)$ early after HIV- 1 seroconversion ( $\leq 1$ year). The viral traits tested were viral replicative capacity, co-receptor usage, and genomic variation. Host $\mathrm{CD}^{+}{ }^{\mathrm{T}}$-cell responses, humoral activity, and HLA immunogenetic markers were also determined.

Results: Our data demonstrate an unusual convergence of highly pathogenic HIV-1 strains in rapid progressors. Compared with standard progressors, rapid progressor viral strains show higher in-vitro replicative capacity $(81.5$ vs. $67.9 \% ; P=0.025)$ and greater $X 4 / \mathrm{DM}$ co-receptor usage $(26.3$ vs. $2.8 \% ; P=0.006)$ in early infection. Limited or absent functional HIV-1 CD ${ }^{+} \mathrm{T}$-cell responses and neutralizing activity were measured in rapid progressors. Moreover, the increase in common HLA allele-restricted $\mathrm{CD} 8^{+} \mathrm{T}$-cell escape mutations in rapid progressors acts as a signature of uncontrolled HIV-1 replication and early impairment of adaptive cellular responses.

Conclusion: Our data support a dominant role for viral factors in rapid progressors. Robust HIV-1 replication and intrinsic viral properties limit host adaptive immune responses, thus driving rapid disease progression.

(C) 2014 Wolters Kluwer Health | Lippincott Williams \& Wilkins

AIDS 2014, 28:000-000

Keywords: HIV-1, host factors, pathogenesis, rapid progressors, viral factors

\footnotetext{
${ }^{a} A$ IDS Research Institute -IrsiCaixa-, Institut d'Investigació en Ciències de la Salut Germans Trias i Pujol, Universitat Autònoma de Barcelona, Badalona, Spain, ${ }^{b}$ Institute of Microbiology, University Hospital Center and University of Lausanne, Lausanne, 'University Clinic of Infectious Diseases, University Hospital Bern and University of Bern, Bern, Switzerland, Universidad Complutense de Madrid, Madrid, Spain, ${ }^{e}$ Monogram Biosciences, South San Francisco, California, USA, ${ }^{\mathrm{f} C e n t r e} \mathrm{~d}^{\prime}$ Estudis Epidemiològics sobre les Infeccions de Transmissió Sexual i Sida de Catalunya (CEEISCAT), Badalona, ${ }^{\mathrm{g}}$ Banc de Sang i Teixits, hUniversitat de Vic, and 'Institució Catalana de Recerca i Estudis Avançats (ICREA), Barcelona, Spain.

Correspondence to Julia G. Prado, PhD, AIDS Research Institute - IrsiCaixa - Hospital Universitari Germans Trias i Pujol, Crta Canyet S/N, 08916 Badalona, Barcelona, Spain.

Tel: +3493465 6374 ext 171; e-mail: jgarciaprado@irsicaixa.es

* Judith Dalmau and Margalida Rotger contributed equally to the writing of this article.

Received: 18 December 2013; revised: 26 March 2014; accepted: 27 March 2014.
}

ISSN 0269-9370 @ 2014 Wolters Kluwer Health | Lippincott Williams \& Wilkins Copyright (C) Lippincott Williams \& Wilkins. Unauthorized reproduction of this article is prohibited. 


\section{Introduction}

The clinical outcome of HIV-1-infected patients varies considerably. In the absence of antiretroviral therapy, the median time to development of AIDS has been estimated to vary from 7.7 to 11.0 years, depending on age at seroconversion [1]. Nevertheless, some individuals are able to control HIV-1 replication for longer periods (HIV-1 controllers and elite controllers), whereas others progress quickly to AIDS or meet the criteria for initiation of antiretroviral treatment within the first 3 years after seroconversion (rapid progressors). Thus, research into extreme phenotypes can provide us with invaluable data on the contribution of viral and host factors to HIV-1 disease outcome [2-4].

Most studies on extreme HIV-1 phenotypes have focused on HIV-1 controllers and elite controllers as models for defining the correlates of protective immunity and control of viral replication $[3,5]$. The study of rapid progressors, on the contrary, has proved particularly challenging, because identification of this phenotype requires a reliable estimation of the seroconversion date within a small observation window [4]. This limitation is even more remarkable if we consider that multiple biological samples need to be taken for analysis. Consequently, very few core studies have analysed rapid progressors, and the data reported thus far are heterogeneous [6-8]. Nevertheless, the factors contributing to rapid disease progression must be uncovered to improve the clinical management of these individuals.

The present study is based on a previous case report study analysing extremely severe progression of HIV-1 infection [7] and attempts to broadly characterize rapid progressors in functional terms by assessing viral and host factors. Our objective was to provide new insights into the causes of rapid disease progression. We recruited an unprecedentedly large group of rapid progressors and systematically compared them to HIV-1-infected patients with average disease progression. Our selection criteria for rapid progressors were based on the reduction in $\mathrm{CD} 4^{+} \mathrm{T}$-cell counts to less than $350 \mathrm{cells} / \mu \mathrm{l}$ within 3 years after documented seroconversion in the absence of antiretroviral treatment, as previously validated by our genomic studies [4].

Our findings reveal a profile of HIV-1-specific traits (e.g. replicative capacity, co-receptor usage, and virus variability) that is common to rapid progressors. Additional differences between groups were observed in cellular and humoral immune profiles and in HLA class I immunogenetics.

\section{Material and methods}

\section{Cohort description and study groups}

We established two progression cohorts: one from IrsiCaixa (Barcelona, Spain) and one from the Swiss
HIV Cohort (Lausanne, Switzerland). The cohorts were defined based on an HIV-1 seroconversion window of less than 1 year and documented negative and positive serology result. Disease progression stage was established during the 3 years after seroconversion (Fig S1, http:// links.lww.com/QAD/A517). Patients in the first cohort (CoRP, $n=106$ ) had two or more CD4 ${ }^{+}$T-cell counts below 350 cells $/ \mu 1$ with no subsequent increase in the absence of antiretroviral treatment. Individuals with average progression or standard progressors (CoSP, $n=84)$ maintained $\mathrm{CD}^{+}{ }^{+}$T-cell counts above 350 cells $/ \mu$ l over the same period in the absence of antiretroviral treatment. From these two cohorts, we selected two study groups: the rapid progressors $(n=46)$ and the standard progressors $(n=46)$. These groups were selected based on clinical data and sample availability early after HIV-1 seroconversion at baseline (median $\leq 1$ year) and a median of 1.5 years from initial sampling postbaseline. Samples were distributed and prioritized according to functional analysis (for details, see Fig S1, http://links.lww.com/QAD/A517). The characteristics of both groups are summarized in Table 1. Differences in the mode of HIV-1 transmission between the groups were due to an increase in the number of intravenous drug users (IVDUs) in rapid progressors. Consistency of the results was demonstrated by sensitivity analyses in all data sets regardless of the presence of IVDUs in rapid progressors. The project was approved by the institutional review boards of Hospital Germans Trias i Pujol and the University Hospital of Lausanne. Participants gave their written informed consent to participate.

\section{HIV-1 isolation, replicative capacity, and co-receptor usage}

Viral isolates were obtained from plasma samples using anti-CD44 beads (Miltenyi Biotec, Germany [7,9]. Viral isolates from cryopreserved PBMCs were obtained by co-culture as described in reference [7]. Virus replicative capacity was determined for all isolates. Viral growth was monitored by p24 ELISA in supernatants (Perkin Elmer, Spain), and replicative capacity was calculated by the slope of $\mathrm{p} 24$ production in the exponential growth phase [10]. Tropism from viral isolates was measured in U87immortalized cell lines expressing CCR 5 or CXCR 4 [11]. Virus tropism was assessed in parallel in plasma samples using the enhanced sensitivity tropism assay (ESTA).

\section{HLA typing and assessment of HIV-1-specific $\mathrm{CD8}^{+} \mathrm{T}$-cell responses}

High-resolution HLA class I typing for alleles A, B, and $\mathrm{Cw}$ was performed by sequence-based typing methods. Comprehensive HIV-1 epitope screening of optimal responses was carried out using the interferon- $\gamma$ (IFN- $\gamma$ ) ELISpot assay in a subset of patients, as previously described [12] (Table S1, http://links.lww.com/QAD/ A517). Wells were considered positive if they contained at least 50 spot-forming cells per $10^{6}$ PBMCs above the 
Table 1. Characteristics of the rapid and standard progressors included in the study.

\begin{tabular}{|c|c|c|c|}
\hline Male sex $[n(\%)]$ & $35(76.1)$ & $43(93.5)$ & 0.02 \\
\hline Age at diagnosis of HIV-1 infection, median (IQR) (years) & $31(26-37)$ & $30(26-35)$ & 0.48 \\
\hline White ethnicity $[n(\%)]$ & $39(84.8)$ & $32(71.1)$ & 0.08 \\
\hline Mode of transmission $[n(\%)]$ & & & 0.002 \\
\hline Heterosexual & $12(26.1)$ & $5(10.9)$ & \\
\hline MSM $^{\mathrm{a}}$ & $20(43.5)$ & $37(80.4)$ & \\
\hline$I_{V D U}{ }^{b}$ & $10(21.7)$ & $1(2.2)$ & \\
\hline Other and NA & $4(8.7)$ & $3(6.5)$ & \\
\hline Viral subtype B [n (\%)] & $38(86.4)$ & $43(93.5)$ & 0.11 \\
\hline Baseline CD4 ${ }^{+}$T-cell count ${ }^{\mathrm{C}}$ [median (IQR)] & $385(245-508)$ & $658(442-804)$ & $<0.0001$ \\
\hline Baseline viral load ${ }^{\mathrm{d}}\left[\log _{10}\right.$ copies/ml, median (IQR)] & $4.88(4.48-5.51)$ & $4.35(3.75-4.99)$ & 0.02 \\
\hline Viral load set point ${ }^{\mathrm{e}}\left[\log _{10}\right.$ copies/ml, median (IQR)] & $4.73(4.28-5.21)$ & $4.10(3.22-4.37)$ & $<0.0001$ \\
\hline Mean $\mathrm{CD}^{+}{ }^{+}$-cell count in the absence of $\mathrm{ART}^{f}(\mathrm{IQR})$ & $317.3(216.1-393.7)$ & $615.3(518.8-713.6)$ & $<0.0001$ \\
\hline
\end{tabular}

IQR, inter-quartile range; IVDU, intravenous drug user; NA, not available.

${ }^{\mathrm{a} M S M}$.

${ }^{\mathrm{b}}$ Intravenous drug users.

${ }^{\mathrm{C}}$ Baseline $\mathrm{CD} 4^{+}{ }^{\mathrm{T}}$-cell count was available for 37 rapid progressors and 30 standard progressors.

dBaseline viral load was available for 34 rapid progressors and 28 standard progressors.

eViral load set point was defined by the level of circulating virus in plasma during the non-symptomatic phase preceding progression to AIDS (steady stage). Data were available for 35 rapid progressors and 37 standard progressors.

${ }^{\mathrm{f}} \mathrm{Mean} \mathrm{CD} 4^{+} \mathrm{T}$-cell count was defined by the mean of the levels of $\mathrm{CD} 4^{+}$cell counts during the non-symptomatic phase preceding progression to AIDS (steady stage). Data were available for 43 rapid progressors and 39 standard progressors.

background level $(2 \times$ mean $+3 \times \mathrm{STD})$. The frequency of HIV-1-specific responses was calculated as follows: [number of positive responses/number of optimal peptides tested] $\times 100$. The frequency of response was corrected by the number of optimal peptides tested per sample.

\section{Evaluation of viral susceptibility to neutralization and plasma HIV-1-neutralizing activity}

Neutralization experiments were performed using the PhenoSense HIV-1 neutralization assay. To determine virus sensitivity to neutralization in rapid progressors and standard progressors, Env-pseudotyped viruses from both groups were tested against a panel of broadly neutralizing antibodies (bNAbs) (b12, 4E10, 2F5, 2G12). Neutralization values are expressed as the concentration of $\operatorname{IgG}$ that reduces infectivity by $50 \%\left(\mathrm{IC}_{50}\right)$. Heterologous neutralizing activity of plasma was tested against a standard panel of HIV-1 isolates. The reference panel viruses NL4-3, SF162, and JR-CSF were included as positive controls and aMLV was used as specificity control. Neutralizing activity was also tested in a panel of autologous and heterologous Env-pseudotyped recombinant viruses obtained from plasma samples [13]. Neutralizing antibody titres in plasma samples were calculated as described in reference [14].

\section{HIV-1 sequencing and variation at optimal $\mathrm{CD8}^{+}$ T-cell epitopes}

Partial HIV-1 genome sequencing was carried out for Gag and Pol genes in plasma samples from 40 patients (22 rapid progressors and 18 standard progressors). Gag and Pol genes were amplified from viral RNA by one-step RT-PCR and nested PCR. Virus subtype was assigned using the REGA HIV-1 subtyping tool in Gag and Pol sequences. Deep sequencing of Gag covering the TW10 region in RP52 was carried out in total viral RNA from plasma or primary viral isolates, as previously described [7].

Optimal HIV-1 CD8 ${ }^{+}$T-cell epitopes were tested based on the best-defined $\mathrm{CD}^{+}$epitope summary from the Los Alamos Molecular Immunology Database. HIV-1 variation at the optimal $\mathrm{CD}^{+}$T-cell epitopes was determined in Gag and Pol viral sequences matched to patients' HLA class I alleles with four-digit resolution. HIV-1 variation at optimal epitopes was defined using the EPIMAP tool (available upon request), which allowed fuzzy matching with up to three substitutions per HIV$1_{\text {HXB2 }}$ epitope and the HLA-I restriction element. The frequency of virus variation was defined as the ratio of total mismatches found at optimal epitopes to the number of total optimal epitopes found per sequence.

\section{Statistical analysis}

For the clinical group comparison, $P$-values were calculated using the Mann-Whitney and Fisher's exact test. The relationship between $\mathrm{CD} 4^{+} \mathrm{T}$-cell count and replicative capacity was assessed using the Spearman's correlation coefficient. The statistical significance of the HLA class allelic distribution was reported after correcting for multiple comparisons using the Benjamin and Hochberg procedure. All analyses were performed using SAS 9.3.

\section{Results}

HIV-1 strains from rapid progressors are highly replicative and have greater X4/DM co-receptor usage

Virus replicative capacity and co-receptor usage are essential for establishing a productive HIV-1 infection 
when transmission bottlenecks occur and for determining the speed of $\mathrm{CD}^{+}$T-cell depletion during early stages. These viral traits have been widely associated with the pathogenesis and outcome of HIV-1 infection [15-18] and are crucial for evaluation in the context of rapid progression of HIV-1. Thus, we assessed viral replicative capacity and co-receptor usage in rapid progressors and compared them with those of standard progressors in samples obtained early after seroconversion (median $\leq 1$ year).

We measured replicative capacity in vitro in primary isolates from rapid progressors $(n=27)$ and standard progressors $(n=20)$. Our results revealed a significantly higher viral replicative capacity in isolates from rapid progressors than in those from standard progressors in early infection (median 81.5 vs. $67.9 \% ; P=0.025$; Fig. 1a). We then studied the correlation between replicative capacity and clinical parameters as predictors of rapid progression. Our data demonstrate an inverse correlation between replicative capacity and baseline CD4 ${ }^{+}$T-cell counts in the overall study group $[19,20]$. In addition, a complementary analysis by disease status revealed the specific contribution of the highest replicative virus to the significant association between replicative capacity and baseline $\mathrm{CD}^{+}{ }^{-} \mathrm{T}$-cell count

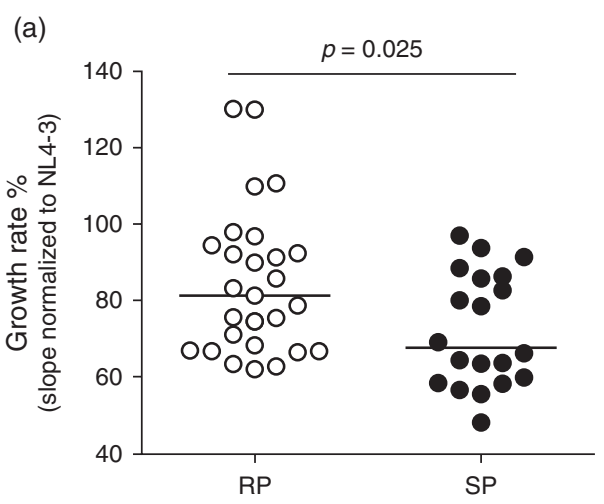

(c)

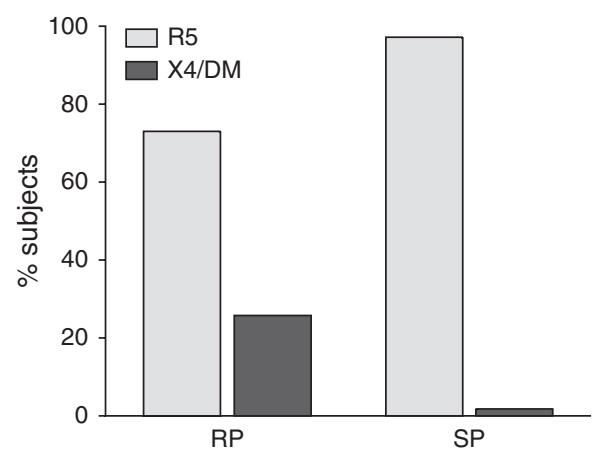

(b)

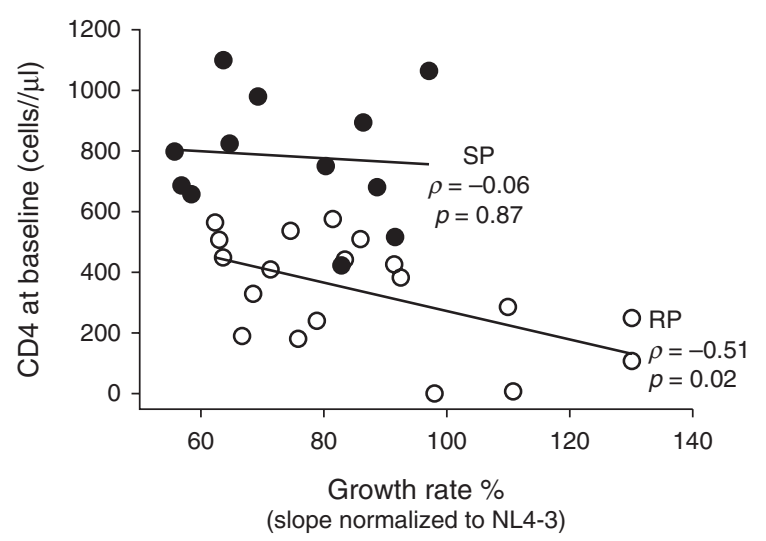

(d)

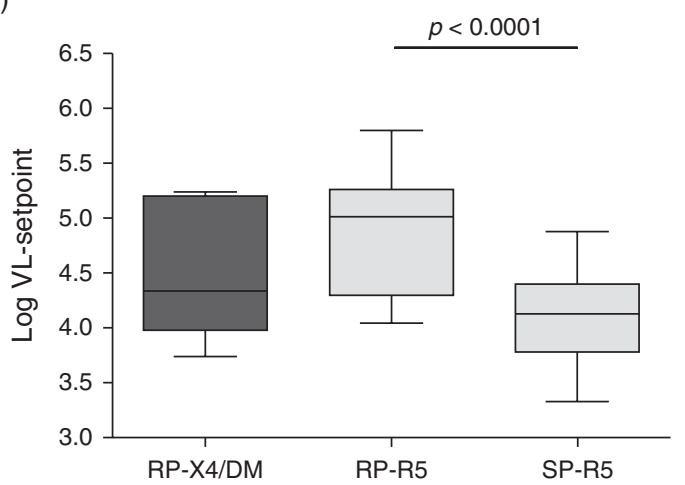

Fig. 1. HIV-1 co-receptor usage and replicative capacity in rapid progressors and standard progressors. (a) Viral replicative capacity from primary isolates in rapid progressors (RPs) and standard progressors (SPs). Replicative capacity was measured in 27 RP and 20 SP at baseline. Experiments were carried out in stimulated PBMC pooled from three seronegative individuals. PBMCs were then infected in duplicate to a multiplicity of infection of 0.001 of each primary isolate in independent cultures for 10 days. Variation between replicates was low. Viral growth was measured every 3 days based on p24 production, and replicative capacity was calculated as the slope in the exponential growth phase for each isolate. Values were normalized to the slope of HIV-1 ${ }_{\text {NL4-3 }}$ as $100 \%$. The horizontal line represents median values (the $P$-value was calculated using the Mann-Whitney test). (b) Correlation between baseline $\mathrm{CD}^{+}{ }^{+} \mathrm{T}$-cell counts and replicative capacity. Lines indicate the fit of the data to linear regressions in $27 \mathrm{RP}$ and 20 SP. The Spearman correlation coefficient $(\rho)$ and the associated two-tailed $P$-value were calculated per group. (c) Total frequency of R5 and X4/DM virus in RP and SP. Viral tropism was evaluated in 38 RP and 36 SP by Trofile and U87. Inter-assay concordance was $96 \%$. Discordant results were recorded in only three samples, all of which were R5 by ESTA and X4/DM by U87. We assigned 2 as R5 by ESTA owing to the very low levels of p24 in U87-X4 culture. The other was defined as X4/DM based on U87-X4 positivity and clonal sequences reporting X4/DM usage. Grey bars represent R5 virus and black bars X4/DM tropic virus. (d) Viral load set point stratified by virus co-receptor usage. The box and whiskers plot shows median values and 10th-90th percentiles in $33 \mathrm{RP}$ and $30 \mathrm{SP}$. The $P$-values were calculated using the Mann-Whitney test. Only significant $P$-values $(P<0.05)$ are shown. ESTA, enhanced sensitivity tropism assay. 
(Fig. 1b, rapid progressors; Spearman's $\rho=-0.51$, $P=0.002)$.

We also analysed virus co-receptor usage in 74 samples (38 rapid progressors and 36 standard progressors) using the ESTA and the U87 phenotypic assay; inter-assay concordance was $96 \%$. We found a previously unreported increase in X4/DM co-receptor usage in association with rapid progression $(26.3 \%$ rapid progressors vs. $2.8 \%$ standard progressors; $P=0.006$ ) (Fig. 1c). These results indicate the contribution of $\mathrm{X} 4 / \mathrm{DM}$ virus to the superior replicative capacity in a subset of rapid progressors. However, despite the increase in X4/DM distribution, the remaining $73.7 \%$ of rapid progressors carried R5 variants. These R5 variants were associated with higher plasma viraemia $(P<0.001$; Fig. $1 \mathrm{~d})$, thus supporting the pathogenic properties of the $\mathrm{R} 5$ virus in rapid progressors.

Taken together, these results demonstrate the contribution of virus replicative capacity and X4/DM co-receptor usage to $\mathrm{CD} 4^{+} \mathrm{T}$-cell depletion and rapid disease progression.

\section{Rapid progressors have weak or absent anti-HIV-1 cellular and humoral adaptive responses}

Once the viral traits that prevail in HIV-1 rapid progressors were established, we investigated the contribution of host immune factors to rapid disease outcome. Therefore, we extended our comparative analyses to cellular and humoral functional markers in our study groups. We focused on virus-specific $\mathrm{CD} 8^{+}$T-cell responses, virus susceptibility to neutralization, and plasma-neutralizing activity as key components of the initial adaptive immune responses against HIV-1.

We measured HIV-1-specific CD8 ${ }^{+}$T-cell responses in a subgroup of 17 rapid progressors and 13 standard progressors in a comprehensive panel of optimally defined HIV-1 $\mathrm{CD}^{+}{ }^{+}$T-cell epitopes. Our results demonstrated weak or even absent HIV-1-specific $\mathrm{CD}^{+}{ }^{+}$-cell responses in rapid progressors when compared to standard progressors at baseline (mean $2.58 \%$ rapid progressors vs. $10.45 \%$ standard progressors; $P=0.004$ ) (Fig. 2a). These differences in early infection were maintained when responses were tested later in infection (post-baseline: mean $1.34 \%$ rapid progressors vs. $8.29 \%$ standard progressors; $P=0.005$ ) (Fig. 2a), with no improvement or recovery despite normalization of $\mathrm{CD}^{+}{ }^{-}$T-cell levels after initiation of treatment in rapid progressors.

We also evaluated humoral responses by measuring virus susceptibility to neutralization and plasma-neutralizing activities across groups. No differences between rapid progressors and standard progressors were observed regarding the sensitivity of HIV-1 Env-pseudotyped virus against a panel of bNAbs (Fig. 2b). Moreover, plasma-neutralizing activity in 12 rapid progressors and 32 standard progressors was evaluated against a panel of viral isolates with a wide range of susceptibilities to neutralization (Table S2, http://links.lww.com/QAD/ A517). Even though a consistent trend towards lower IC I0 $_{50}$ values was observed in rapid progressors, the results did not reach statistical significance (Fig. 2c). Similarly, no differences were found between groups in total heterologous plasma-neutralizing activity (Fig. 2d and Table S3, http://links.lww.com/QAD/A517). Furthermore, autologous plasma neutralization was generally low against contemporaneous virus and only increased in standard progressors over time (rapid progressors vs. standard progressors; $P=0.019$ ) (Fig. 2e). By contrast, autologous neutralizing titres against ancestral viruses were higher in both groups [rapid progressors $(P=0.024)$ and standard progressors $(P<0.0001)]$, but with a lower increase in the rapid progressors within the same period (Fig. 2e).

These data demonstrate the lack of early anti-HIV-1 adaptive host immune response in rapid disease progression. Weak or absent specific $\mathrm{CD} 8^{+} \mathrm{T}$-cell responses, together with limited neutralizing activity, define a common dysfunctional immune phenotype in rapid progressors.

\section{Presence of HIV-1 variants adapted to common HLA class I alleles is associated with rapid disease progression}

The high rate of initial virus adaptation to or escape from HLA class I molecules is a major limitation for the early control of HIV-1 by CD8 ${ }^{+}$T-cell responses [21]. Thus, transmission of HLA-adapted variants has been associated with rapid progression [2], even in individuals with protective HLA alleles $[22,23]$. Therefore, in order to understand the lack of early anti-HIV-1 $\mathrm{CD}^{+}$T-cell responses in rapid progressors, it is critical to identify the contribution of HIV-1 adaptation to or escape from HLA class I immunogenetics.

Initially, to define the contribution of HLA-adapted strains to rapid progression, we focused on patients with protective HLA class I alleles (HLA-B*58 and $\mathrm{B} * 27$ ) and rapid progression. We identified one HLA-B*27 rapid progressors and three HLA-B*58 rapid progressors. We evaluated the existence of adapted virus to protective KK10 and TW10 Gag epitopes. As a result, we found immune escape variants at positions K264R in KK10 and $\mathrm{T} 242 \mathrm{~N}$ in TW10 in all rapid progressors. By contrast, no immune escape was observed in two HLA-B*27 standard progressors within the same period of infection (Fig. 3a). Additionally, Gag K264R and T242N mutations known to cause viral replication defects [24,25] appeared alongside compensatory changes in replication at positions S173 for K264R and I223/G248 for T242N in rapid progressors (Fig. 3a). Development of such a 


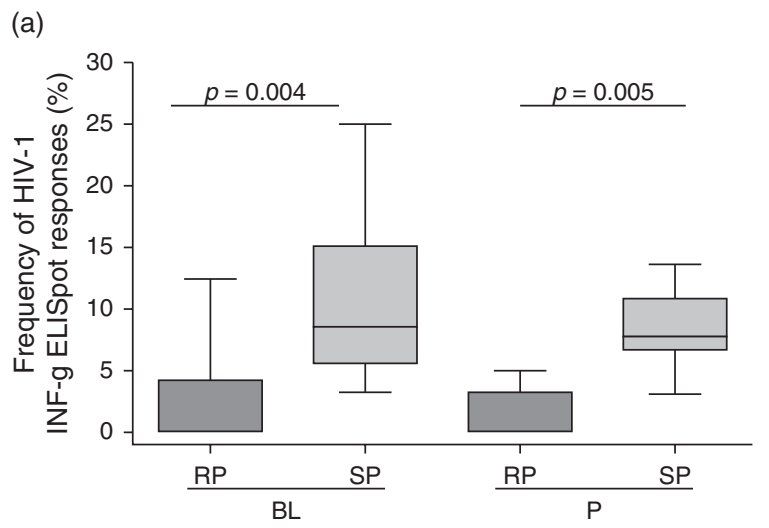

(b) bNAb

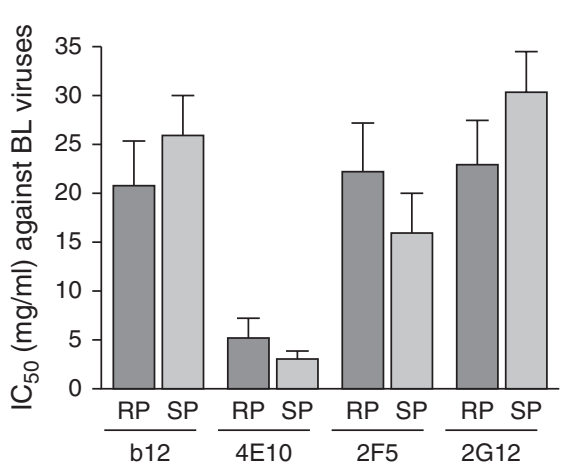

(d) Heterologous neutralization

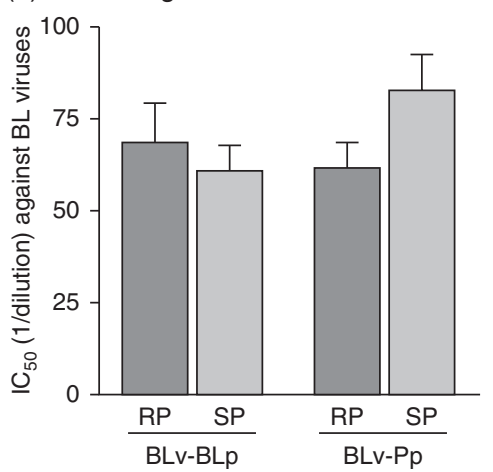

(c) Virus panel

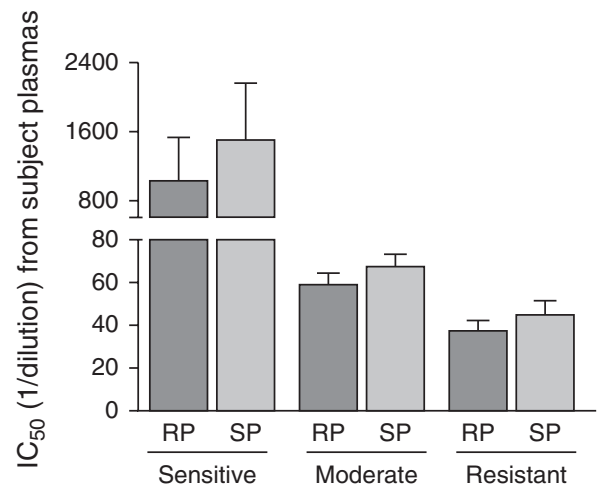

(e) Autologous neutralization

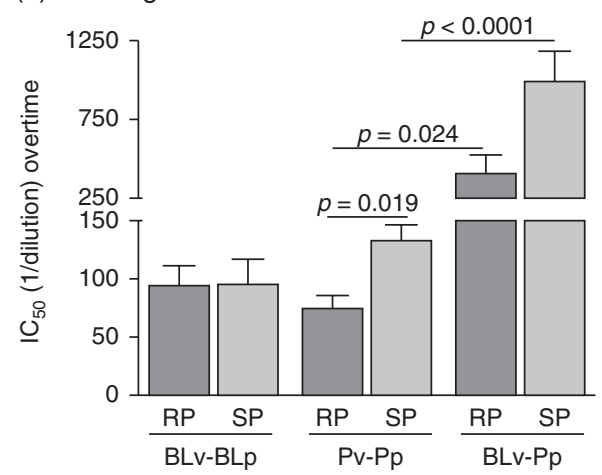

Fig. 2. HIV-1-specific $\mathrm{CD8}^{+}$T-cell responses, virus sensitivity to neutralization and plasma-neutralizing activity in rapid progressors and standard progressors. (a) Frequency of IFN- $\gamma$ HIV-1-specific CD8 ${ }^{+}$T-cell responses at baseline (BL) and postbaseline $(\mathrm{P})$ were measured in PBMCs against a panel of optimally defined HLA class I HIV-1 epitopes in 17 rapid progressors (RPs) and 13 standard progressors (SPs). The box and whiskers plot shows median values and 10 th and 90th percentiles. The $P$-values were calculated using the Mann-Whitney test. Only significant $P$-values $(P<0.05)$ are shown. (b) Sensitivity to neutralization of Env-pseudotyped virus derived from the plasma of 24 RP and 31 SP against a panel of broadly neutralizing antibodies (bNAbs) including b12, 4E10, 2F5, and 2G12. (c) Heterologous plasma-neutralizing activity at baseline against a standard panel of viral isolates from various subtypes and with various ranges of susceptibilities to neutralization (sensitive, moderate, and resistant). (d) Heterologous cross-reactive virus-neutralizing activity in plasma samples, at baseline (BLp) and post-baseline (Pp), against a panel of baseline patient-derived Env-pseudotyped viruses (BLv). (e) Autologous neutralizing activity in plasma and Envpseudotyped virus pairs. All $P$-values were calculated using the Mann-Whitney test. Only significant $P$-values $(P<0.05)$ are represented. BLp, baseline plasma; BLv, baseline virus; Pp, post-baseline plasma; Pv, post-baseline virus. 
(a)

\begin{tabular}{|c|c|c|}
\hline & & KK10 \\
\hline CONSENSUSB & $\underset{173}{\text { PEVIPMFSALSEGAT }}$ & $\begin{array}{l}\text { IPVGEIYKRWIILGLNKIVRMY } \\
\qquad 264 \quad 268\end{array}$ \\
\hline RP26 B*27 & $\ldots \ldots$. . . . & . K...M. \\
\hline SP14 B*27 & $\ldots \ldots \ldots \ldots \ldots$ & $\ldots \ldots$ \\
\hline $\mathrm{P} 21 \mathrm{~B}^{*} 27$ & $\ldots \ldots \ldots \ldots$ & $\ldots \ldots \ldots \ldots \ldots \ldots$ \\
\hline
\end{tabular}

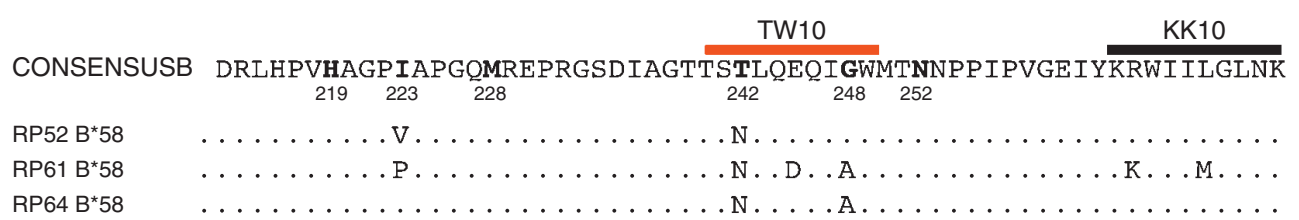

(b)

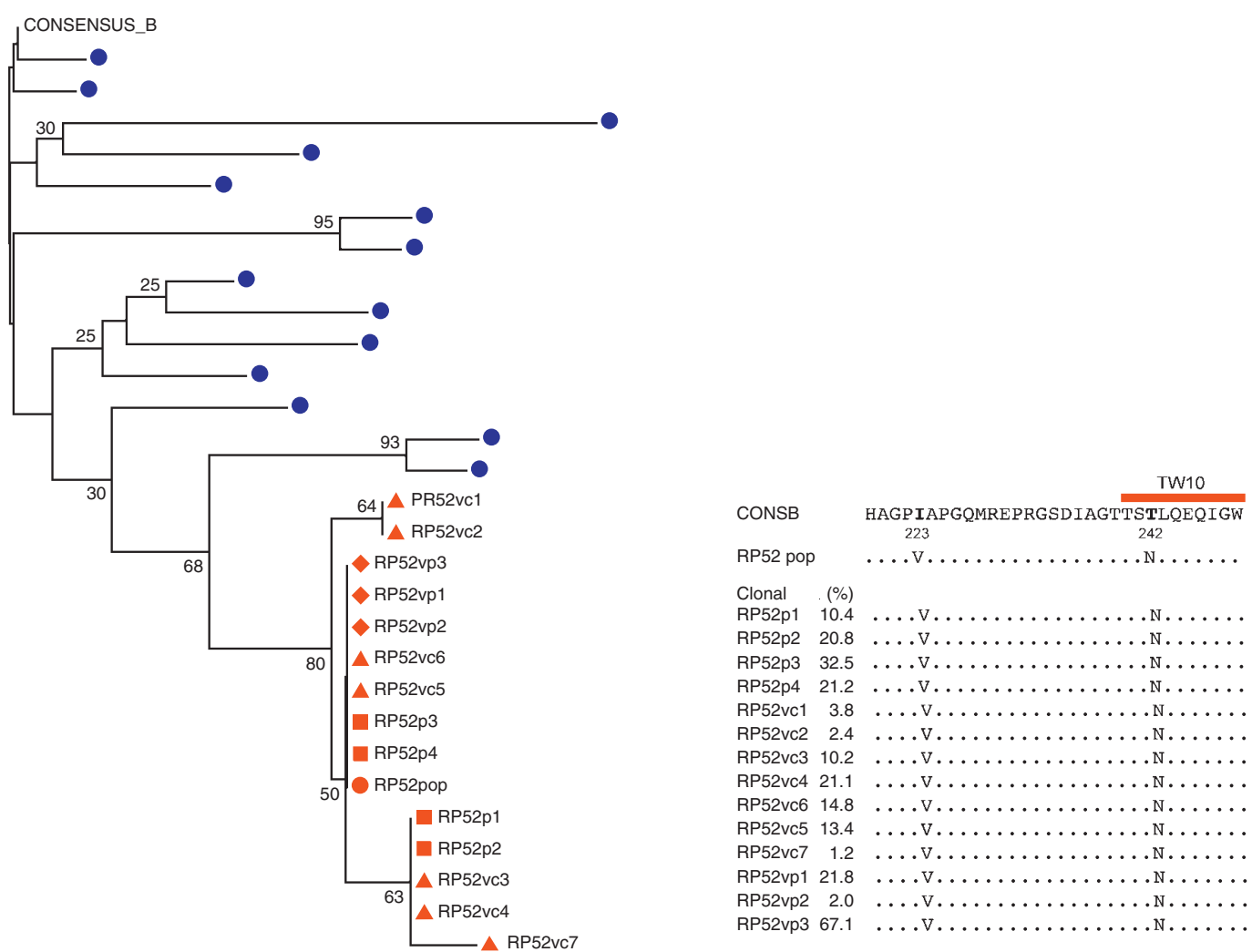

Fig. 3. HIV-1 strains adapted to HLA-B $* 58$ and $B * 27$ alleles in rapid progressors at Gag TW10 and KK10 protective epitopes. Gag viral sequences were obtained from plasma samples in an HLA-B*27 RP (RP26) and 3 HLA-B*58 RP (RP52, RP61 and RP64). For comparative purposes, Gag sequences were obtained from two HLA-B*27 SP (SP14, SP21). (a) Comparison of HIV-1 Gag amino acid sequences at KK10 and TW10 epitopes. Epitopes are marked at the top of the sequence with a black line (KK10) or red line (TW10). Letters in bold indicate residues associated with escape in KK10 $(264,268)$ and replicative capacity compensatory sites (173). Similarly, bold letters indicate escape in TW10 (242) and replicative capacity compensatory sites (219, 223, 248, 252). Amino acid numbering is based on the HXB2 sequence. (b) Gag phylogenetic tree in HLA-B*58 RP52 covering the TW10 epitope and the 223 compensatory site at 12 months after seroconversion. The tree was built by the neighbour-joining method based on over 1000 bootstrap replicates. The tree was rooted to the consensus B Gag sequence and edited with MEGA.5. The number at the nodes indicates bootstrap support. Blue dots indicate Gag population sequences from additional RP. Red symbols indicate RP52 Gag sequences. The red dot represents the population sequence (RP52pop). Squares, diamonds, and triangles represent clonal viral sequences. Red triangles and diamonds are clonal viral sequences obtained from primary viral isolates from cells (RP52vc) or plasma (RP52vp). Red squares are clonal viral sequences obtained directly from total plasma (RP52p). The alignment next to the tree shows the clonal populations of TW10 escape variants in RP52. The frequency of the variant in the total population is expressed as a percentage next to each clonal sequence. RP, rapid progressors; SP, standard progressors. 
complex mutational pattern is rare in early infection $[23,26,27]$ and likely represents a marker of HIV-1 transmission events. This observation is reinforced by the presence of KK10 escape mutants in non-B*27 rapid progressors (Fig. 3a) and by the homogeneous clonal populations of TW10 escape mutants found in an HLAB*58 rapid progressors (Fig. $3 \mathrm{~b}$ ). Overall, these findings are consistent with the low level of viral genetic variability described in recent infection and probably identify transmission events in rapid progression.

With the aim of generalizing previous observations to specific immunogenetic profiles in rapid progressors, we performed an exploratory analysis of HLA class I distribution in $122 \mathrm{HIV}-1$-infected individuals (74 rapid progressors and 48 standard progressors). Our data demonstrate an increase in the frequency of common Caucasian HLA haplotypes, including HLA-A*02:01, $\mathrm{B} * 07: 02$, and $\mathrm{B} * 08: 01$ in rapid progressors $(75.6 \%$ rapid progressors vs. $50 \%$ standard progressors) to the detriment of protective ones $(6.76 \%$ rapid progressors vs. $22.9 \%$ standard progressors) Fig. 4a. In addition, a modest increase was observed in risk alleles. Similar trends across data analyses were supported by individual HLA type analyses (Table S4, http://links.lww.com/QAD/A517).

In order to evaluate the contribution of virus adaptation or escape to the immunogenetics of rapid progressors, we focused on HLA-A*02:01 carriers because of their higher prevalence in the study population. Thus, we determined HIV-1 variation at the immunodominant $A * 02: 01-$ restricted SL9-Gag epitope. We compared HIV-1 SL9Gag variation in HLA-A*02:01-positive individuals $(A * 02: 02+v e)$ early after seroconversion (Fig. 4b). Our data demonstrated preferential variation at position $3(\mathrm{Y} 3 \mathrm{~F})$, but not position 8, of the SL9-Gag epitope in $\mathrm{A} * 02: 01+\mathrm{ve}$ rapid progressors when compared to $\mathrm{A}^{*} 02: 01+$ ve standard progressors $(62.5 \%$ rapid progressors vs. $0 \%$ standard progressors; $P=0.031$ ) (Fig. $4 \mathrm{~b}$ ). These differences revealed a specific increase in SL9 escape variants in rapid progressors. Furthermore, to extend these findings to complete viral proteins, we measured total variation in Gag and Pol regions and limited the analyses to those epitopes recognized by each patient's HLA. Our data indicated a significant increase in total Gag epitope variation in viral strains from rapid progressors when compared to standard progressors (1 rapid progressors vs. 0.63 standard progressors; $P=0.008$ ) (Fig. 4c), but not in Pol. Moreover, these differences in epitope variation were not equally distributed across functional domains in Gag, but significantly accumulated in the $\mathrm{p} 24$ region $(0.87$ rapid progressors vs. 0.35 standard progressors; $P=0.0084$ ) (Fig. $4 \mathrm{~d}$ ). These results support the association between HLA class I adaptation or escape of HIV-1 variants and rapid disease progression. This association is marked by a high prevalence of common Caucasian haplotypes and higher mutational changes against p24 epitopes.

\section{Discussion}

The time required for the development of AIDS after HIV-1 infection is associated with the temporal equilibrium between viral and host factors. This dynamic interplay can be disrupted and present as extreme disease that either contributes to prolonged periods of HIV-1 control or drives rapid disease progression. In this context, rapid progression remains one of the least understood extreme HIV-1 phenotypes, yet it must be understood if we are to tackle the epidemic. Consequently, the present study overcomes previous study limitations through the identification and functional characterization of the largest group of rapid progressors to date. For the first time, we provide evidence of the contribution of intrinsic viral properties to rapid disease progression in a study group.

Our data demonstrate how viral factors, including robust HIV-1 replication, higher X4/DM co-receptor usage, and an increased number of common HLA footprints, could lead to uncontrolled virus replication. Such a robust replicative profile will likely limit the generation of adaptive responses and precipitate rapid disease progression. The pathogenic HIV-1 strains found in rapid progressors are rare at the onset of infection, and discrepancies in viral factors are most apparent with regard to viral tropism. Thus, the reported prevalence of X4/ DM virus ranged from 5 to $10 \%$ in primary infection $[17,28]$, compared with above $25 \%$ in rapid progressors. However, differences in the selection criteria according to rapid disease progression might partially account for the divergence between studies. Although X4/DM viruses are more pathogenic than $\mathrm{R} 5$ viruses [29], $\mathrm{R} 5$ variants in rapid progressors were equally associated with high replicative capacity and viral load set point. Therefore, these data highlight a previously unnoticed contribution of R5 strains to disease progression.

Intrinsic viral properties may also contribute to the limited adaptive immunity found in rapid progressors, which is key to controlling HIV-1 replication upon transmission. We observed defective HIV-1-specific $\mathrm{CD}^{+}$cellular responses and no major role of neutralizing antibodies, as previously reported $[30,31]$. However, the generalized static immune phenotype in rapid progressors does not reflect a pre-existing immunodeficiency [2], but rather an outstanding loss of $\mathrm{CD}^{+}{ }^{-} \mathrm{T}$ cells in primary infection. The dramatic loss of $\mathrm{CD}^{+} \mathrm{T}$ cells will irreversibly affect $\mathrm{CD} 4^{+}$homeostasis and thus compromise $\mathrm{CD} 4^{+} \mathrm{T}$-cell helper responses and $\mathrm{CD} 8^{+} \mathrm{T}$-cell [32] and $\mathrm{B}$-cell function $[33,34]$. In addition, subsequent limited anti-HIV-1-adaptive immunity established early on could add to the lack of control of viral replication and disease progression.

In this study, we observed early disruption of host immunity in favour of viral replication. This phenomenon 
(a)

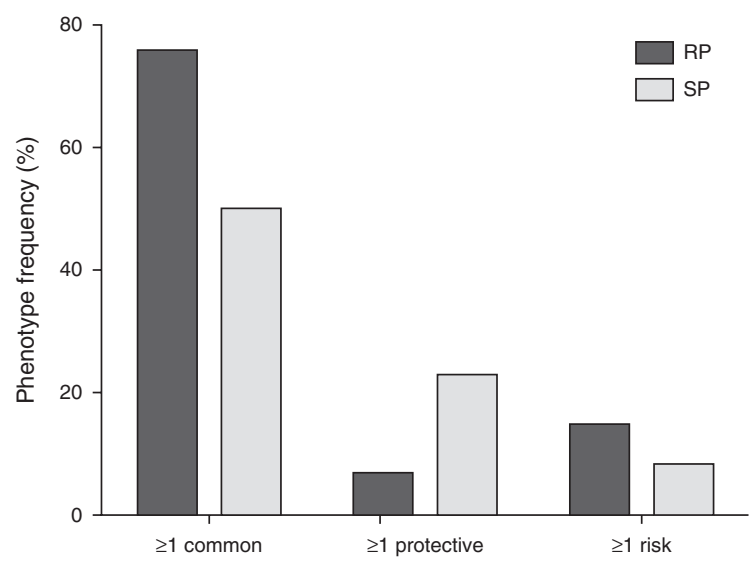

(b)
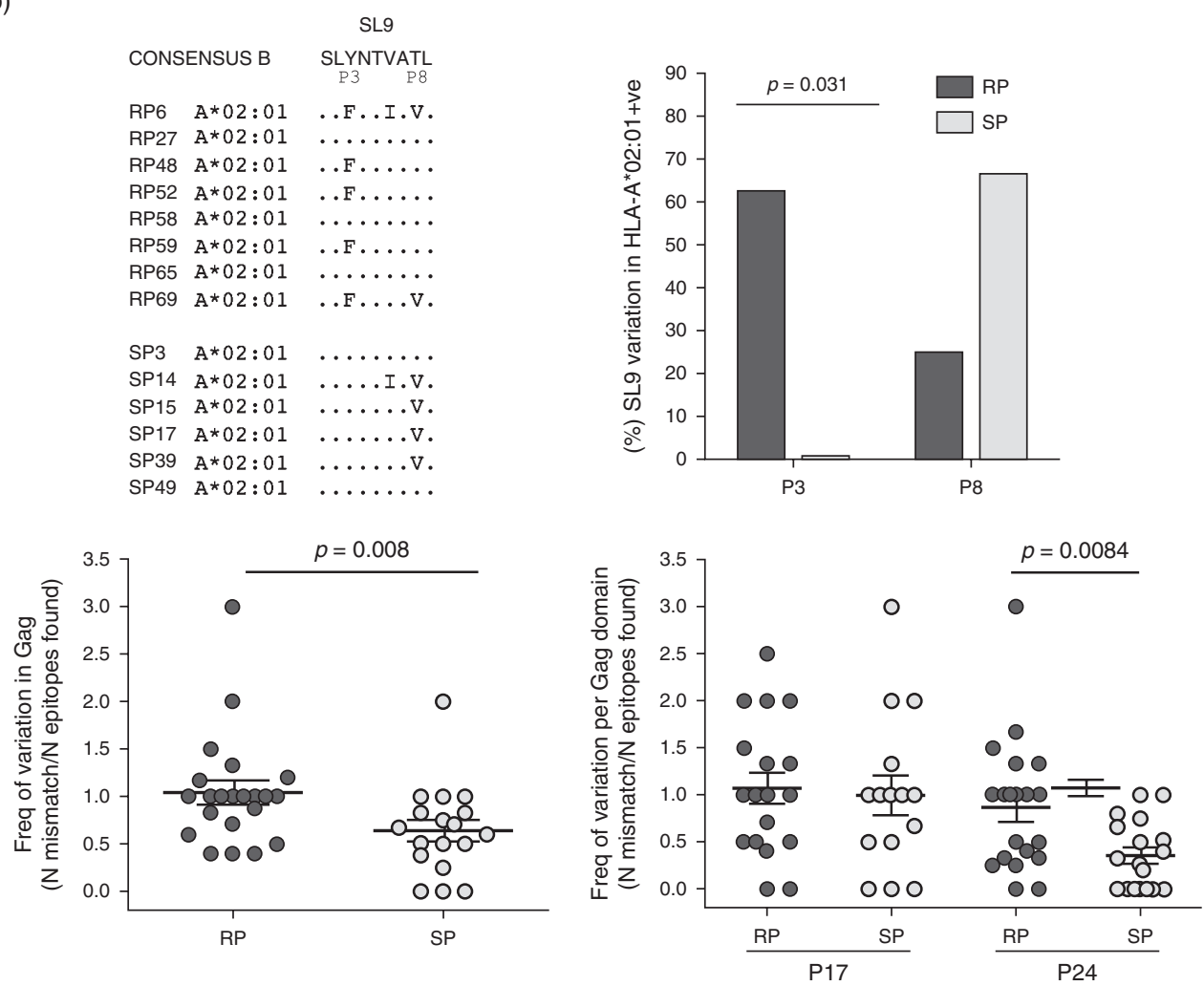

Fig. 4. HLA class I immunogenetics and HIV-1 variation at HLA-matched epitopes in rapid progressors and standard progressors. (a) Phenotypic frequency (number of patients with a defined haplotype/number of total individuals in the group $\times 100$ ) in pooled analyses for HLA class I alleles. Patients in groups (74 RP and 48 SP) were included if they had at least one common allele $\left(A^{*} 02: 01, B^{*} 07: 02\right.$ and $\left.B * 08: 01\right)$, a protective allele $(B * 57: 01, B * 14: 01$ and $B * 27: 05)$, or a risk allele $(B * 35: 02$, $B * 35: 03, B * 35: 04$ and $B * 53: 01)$. Only those alleles with a frequency above 3\% in the overall population were taken into account for data representation. (b) HIV-1 SL9 epitope alignment in HLA-A*02:01+ve RP and SP; P3 and P8 indicate amino acid sites in SL9. The bar graph shows the frequency of epitope variation at P3 and P8 sites in HLA-A*02:01+ve SP and RP. (c) Frequency of epitope variation in HIV-1 Gag. Partial sequencing was carried out in a total of 40 patients (22 RP and 18 SP). (d) Frequency of HLAmatched epitope variation in HIV-1 Gag per functional domains p17 and p24. Values in (c) and (d) are expressed as mean and SEM. The $P$-values were calculated using the Mann-Whitney test. Only significant $P$-values $(P<0.05)$ are represented. RP, rapid progressors; SP, standard progressors.

seems to be associated with the increase in HLA class I virus adaptation or escape. HIV-1 adapts to the most frequent alleles in a population [21]. Therefore, the probability of transmission of adapted viruses will increase among common HLA carriers, thus reducing the chances for a potent anti-HIV-1 $\mathrm{CD}^{+}$T-cell response [21,35]. Consistent with this hypothesis, our data identified the accumulation of common Caucasian alleles, including 
$\mathrm{A} * 02: 01, \mathrm{~B} * 07: 02$, and $\mathrm{B} * 08: 01$, in more than $75 \%$ of rapid progressors. Our results confirm and extend those of previous studies showing a disadvantage of A2 and B7 supertypes in control of HIV-1 infection [35,36]. According to this immunogenetics profile, the likelihood of having adapted or escaped variants should increase in rapid progression of HIV-1. This observation is consistent with the presence of well adapted and well compensated viruses in HLA-B*58 and HLA-B*27 patients and reinforced by the spread of SL9 escaped variants in HLA-A*02:01-positive rapid progressors. In addition, the overall increase in HIV-1 Gag-p24 mutations matched to HLA class I epitopes in rapid progressors, but not in standard progressors, points to the existence of adapted or escape variants during the first year after seroconversion. Despite the fact that we cannot directly associate specific amino acid changes in Gag-p24 epitopes with HIV-1 escape, multiple intra-epitope variation might interfere with the generation of particularly protective $\mathrm{Gag} \mathrm{CD}^{+}$ T-cell responses [3,37-39]. Although our study demonstrated an increase in HLA class I-adapted HIV-1 variants with rapid progression, we were unable to determine whether viruses were transmitted as homogeneous adapted founder strains or whether escape mutations quickly emerge through the robust replicative profile in acute infection. Nevertheless, the presence of viruses with complex mutational patterns found only at late stages of disease $[23,26,40]$ and homogeneous populations of escape mutants in early infection seem to indicate that transmission events are the origin of these pathogenic strains. Thus, rapid progression could originate from acquisition of HIV-1 from chronically infected untreated individuals [41]. These source patients increase the probability of transmission of X4/DM or pathogenic R5 variants [42], which are fully replicative and adapted to host HLA through long-term viral replication.

The limitations of the present study include the lack of transmission pairs to clarify the contribution of founder viruses to rapid progression. Moreover, ideally, comparisons between groups should be made during acute stages, which might minimize differences at baseline. However, this would reduce the number of study participants and thus make it difficult to draw conclusions. Additionally, availability of biological material was limited owing to the size and heterogeneity of the samples included in some sub-analyses.

In conclusion, our findings suggest that rapid progression results from the acquisition of highly pathogenic strains already adapted to HLA class I alleles. These strains avoid the initial control of HIV-1 replication mediated by adaptive $\mathrm{CD}^{+}$T-cell responses, leading to rapid progression, and might help to explain the increased virulence of HIV-1 observed over the course of the epidemic [43]. Therefore, it would be advisable to develop strategies for early identification of rapid progressors, specific clinical guidelines, and robust prevention policies in order to provide adequate care and to prevent the spread of highly pathogenic viral strains in the population.

\section{Acknowledgements}

We thank all the participants in the study and the clinical team of the Fundació Lluita contra la SIDA. This study was supported by the European Union's FP7/2007-2013 under the 'Collaborative HIV and Anti-HIV Drug Resistance Network (CHAIN)' Project Grant agreement 223131 and the 'Catalonian HIV-1 Vaccine Program' (HIVACAT program). Work at J.M.P.'s laboratory is supported by the Spanish Department of Science, Development and Innovation through grant SAF201021224. Work at A.T.'s laboratory is supported by the Swiss National Science Foundation within the framework of the Swiss HIV Cohort Study (grant \# 33CS30_134277). Work at J.G.P.'s laboratory is supported by grants CP09/ 00279 and PI11/00249 from the FIS-ISCIII. We thank D. Ouchi for statistical support. We thank Z. Brumme for advice on HLA data analysis. We thank G. Fernandez for critical comments on the manuscript.

Author contributions: J.D., M.R., A.T., J.M.P. and J.G.P. were responsible for patient selection, study design, data interpretation, and manuscript writing. P.P. performed experiments on humoral responses. J.D., I.E., M.P., and J.G.P. conceived and performed experiments on virus isolation, co-receptor usage, replication, sequencing, and cellular responses. P.R. developed the EPIMAP tool and reviewed the manuscript. E.P. was responsible for HLA data generation. A.E. provided statistical support and reviewed the manuscript. A.T., C.B., R.P., and B.C. contributed to data interpretation and writing and review of the manuscript. All the authors approved the final manuscript.

\section{Conflicts of interest}

J.G.P. holds a Miguel Servet Contract (MS09/00279) funded by FIS-ISCIII (Spanish Government). The funders had no role in study design, data collection and analysis, decision to publish, or preparation of the manuscript.

The authors have no conflicting financial interests.

\section{References}

1. Time from HIV-1 seroconversion to AIDS and death before widespread use of highly-active antiretroviral therapy: a collaborative re-analysis. Collaborative Group on AIDS Incubation and HIV Survival including the CASCADE EU Concerted Action Concerted Action on SeroConversion to AIDS and Death in Europe. Lancet 2000; 355:1131-1137. 
2. Dalmau J, Puertas MC, Azuara M, Mariño A, Frahm N, Mothe B, et al. Contribution of immunological and virological factors to extremely severe primary HIV type $\mathbf{1}$ infection. Clin Infect Dis 2009; 48:229-238.

3. Pereyra F, Addo MM, Kaufmann DE, Liu Y, Miura T, Rathod A, et al. Genetic and immunologic heterogeneity among persons who control HIV infection in the absence of therapy. J Infect Dis 2008; 197:563-571.

4. Rotger M, Dalmau J, Rauch A, McLaren P, Bosinger SE, Martinez $R$, et al. Comparative transcriptomics of extreme phenotypes of human HIV-1 infection and SIV infection in sooty mangabey and rhesus macaque. / Clin Invest 2011; 121:2391-2400.

5. Pereyra F, Jia X, McLaren PJ, Telenti A, de Bakker PIW, Walker $\mathrm{BD}$, et al. The major genetic determinants of HIV-1 control affect HLA class I peptide presentation. Science 2010; 330:1551-1557.

6. Casado C, Colombo S, Rauch A, Martínez R, Günthard HF, Garcia $S$, et al. Host and viral genetic correlates of clinical definitions of HIV-1 disease progression. PLOS One 2010; 5: e11079.

7. Dalmau J, Codoñer FM, Erkizia I, Pino M, Pou C, Paredes R, et al. In-depth characterization of viral isolates from plasma and cells compared with plasma circulating quasispecies in early HIV-1 infection. PLoS One 2012; 7:e32714.

8. Markowitz M, Mohri H, Mehandru S, Shet A, Berry L, Kalyanaraman $R$, et al. Infection with multidrug resistant, dual-tropic HIV-1 and rapid progression to AIDS: a case report. Lancet 2005; 365:1031-1038.

9. Prado JG, Prendergast A, Thobakgale C, Molina C, TudorWilliams G, Ndung'u T, et al. Replicative capacity of human immunodeficiency virus type $\mathbf{1}$ transmitted from mother to child is associated with pediatric disease progression rate. J Virol 2010; 84:492-502.

10. Prado JG, Honeyborne I, Brierley I, Puertas MC, MartinezPicado J, Goulder PJR. Functional consequences of human immunodeficiency virus escape from an HLA-B $* 13$-restricted CD8+ T-cell epitope in p1 Gag protein. / Virol 2009; 83:10181025.

11. Björndal A, Deng H, Jansson M, Fiore JR, Colognesi C, Karlsson $A$, et al. Coreceptor usage of primary human immunodeficiency virus type 1 isolates varies according to biological phenotype. / Virol 1997; 71:7478-7487.

12. Addo MM, Yu XG, Rathod A, Cohen D, Eldridge RL, Strick D, et al. Comprehensive epitope analysis of human immunodeficiency virus type 1 (HIV-1)-specific T-cell responses directed against the entire expressed HIV-1 genome demonstrate broadly directed responses, but no correlation to viral load. I Virol 2003; 77:2081-2092.

13. Schweighardt B, Liu Y, Huang W, Chappey C, Lie YS, Petropoulos CJ, Wrin T. Development of an HIV-1 reference panel of subtype $B$ envelope clones isolated from the plasma of recently infected individuals. I Acquir Immune Defic Syndr 2007; 46:1-11.

14. Frost SDW, Wrin T, Smith DM, Kosakovsky Pond SL, Liu Y, Paxinos $E$, et al. Neutralizing antibody responses drive the evolution of human immunodeficiency virus type 1 envelope during recent HIV infection. Proc Natl Acad Sci U S A 2005; 102:18514-18519.

15. Ferbas J, Daar ES, Grovit-Ferbas K, Lech WJ, Detels R, Giorgi JV, Kaplan AH. Rapid evolution of human immunodeficiency virus strains with increased replicative capacity during the seronegative window of primary infection. / Virol 1996; 70:72857289.

16. Mohri $\mathrm{H}$, Markowitz $M$. In vitro characterization of multidrug-resistant HIV-1 isolates from a recently infected patient associated with dual tropism and rapid disease progression. I Acquir Immune Defic Syndr 2008; 48:511521.

17. Raymond S, Delobel P, Mavigner M, Cazabat M, Encinas S, Souyris $C$, et al. CXCR4-using viruses in plasma and peripheral blood mononuclear cells during primary HIV-1 infection and impact on disease progression. AIDS 2010; 24:2305-2312.

18. Richman DD, Bozzette SA. The impact of the syncytium-inducing phenotype of human immunodeficiency virus on disease progression. J Infect Dis 1994; 169:968-974.
19. Barbour JD, Hecht FM, Wrin T, Segal MR, Ramstead CA, Liegler TJ, et al. Higher CD4+ T cell counts associated with low viral pol replication capacity among treatment-naive adults in early HIV-1 infection. J Infect Dis 2004; 190:251-256.

20. Daar ES, Kesler KL, Wrin T, Petropoulo CJ, Bates M, Lail A, et al. HIV-1 pol replication capacity predicts disease progression. AIDS 2005; 19:871-877.

21. Kawashima Y, Pfafferott K, Frater J, Matthews P, Payne R, Addo $M$, et al. Adaptation of HIV-1 to human leukocyte antigen class I. Nature 2009; 458:641-645.

22. Crawford $\mathrm{H}$, Lumm W, Leslie A, Schaefer M, Boeras D, Prado $\mathrm{JG}$, et al. Evolution of HLA-B $* 5703$ HIV-1 escape mutations in HLA-B $* 5703$-positive individuals and their transmission recipients. J Exp Med 2009; 206:909-921.

23. Goulder PJ, Brander C, Tang Y, Tremblay C, Colbert RA, Addo $M M$, et al. Evolution and transmission of stable CTL escape mutations in HIV infection. Nature 2001; 412:334-338.

24. Martinez-Picado J, Prado JG, Fry EE, Pfafferott K, Leslie A, Chetty $\mathrm{S}$, et al. Fitness cost of escape mutations in p24 Gag in association with control of human immunodeficiency virus type 1. J Virol 2006; 80:3617-3623.

25. Schneidewind A, Brockman MA, Yang R, Adam RI, Li B, Le Gall $S$, et al. Escape from the dominant HLA-B27-restricted cytotoxic T-lymphocyte response in Gag is associated with a dramatic reduction in human immunodeficiency virus type 1 replication. / Virol 2007; 81:12382-12393.

26. Feeney ME, Tang Y, Roosevelt KA, Leslie AJ, McIntosh K, Karthas $\mathrm{N}$, et al. Immune escape precedes breakthrough human immunodeficiency virus type 1 viremia and broadening of the cytotoxic T-lymphocyte response in an HLA-B27-positive longterm-nonprogressing child. / Virol 2004; 78:8927-8930.

27. Schneidewind A, Brumme ZL, Brumme CJ, Power KA, Reyor LL, $\mathrm{O}^{\prime}$ Sullivan $\mathrm{K}$, et al. Transmission and long-term stability of compensated CD8 escape mutations. J Virol 2009; 83:39933997.

28. Chalmet K, Dauwe K, Foquet L, Baatz F, Seguin-Devaux C, Van Der Gucht B, et al. Presence of CXCR4-using HIV-1 in patients with recently diagnosed infection: correlates and evidence for transmission. J Infect Dis 2012; 205:174-184.

29. Koot M, Keet IP, Vos AH, de Goede RE, Roos MT, Coutinho RA, et al. Prognostic value of HIV-1 syncytium-inducing phenotype for rate of CD4+ cell depletion and progression to AIDS. Ann Intern Med 1993; 118:681-688.

30. Hay CM, Ruhl DJ, Basgoz NO, Wilson CC, Billingsley JM, DePasquale MP, et al. Lack of viral escape and defective in vivo activation of human immunodeficiency virus type 1specific cytotoxic T lymphocytes in rapidly progressive infection. J Virol 1999; 73:5509-5519.

31. Richman DD, Wrin T, Little SJ, Petropoulos CJ. Rapid evolution of the neutralizing antibody response to HIV type 1 infection. Proc Natl Acad Sci U S A 2003; 100:4144-4149.

32. Nakanishi Y, Lu B, Gerard C, Iwasaki A. CD8(+) T lymphocyte mobilization to virus-infected tissue requires CD4(+) T-cell help. Nature 2009; 462:510-513.

33. Lindqvist M, van Lunzen J, Soghoian DZ, Kuhl BD, Ranasinghe $S$, Kranias G, et al. Expansion of HIV-specific T follicular helper cells in chronic HIV infection. / Clin Invest 2012; 122:32713280.

34. Petrovas C, Yamamoto T, Gerner MY, Boswell KL, Wloka K, Smith EC, et al. CD4 T follicular helper cell dynamics during SIV infection. J Clin Invest 2012; 122:3281-3294.

35. Trachtenberg E, Korber B, Sollars C, Kepler TB, Hraber PT, Hayes $\mathrm{E}$, et al. Advantage of rare HLA supertype in HIV disease progression. Nat Med 2003; 9:928-935.

36. Peterson TA, Kimani J, Wachihi C, Bielawny T, Mendoza L, Thavaneswaran S, et al. HLA class I associations with rates of HIV-1 seroconversion and disease progression in the Pumwani Sex Worker Cohort. Tissue Antigens 2013; 81:93-107.

37. Kiepiela P, Ngumbela K, Thobakgale C, Ramduth D, Honeyborne I, Moodley E, et al. CD8+ T-cell responses to different HIV proteins have discordant associations with viral load. Nat Med 2007; 13:46-53.

38. Sáez-Cirión A, Lacabaratz C, Lambotte O, Versmisse P, Urrutia A, Boufassa F, et al. HIV controllers exhibit potent CD8 T cell capacity to suppress HIV infection ex vivo and peculiar cytotoxic T lymphocyte activation phenotype. Proc Natl Acad Sci U S A 2007; 104:6776-6781. 
39. Zuñiga R, Lucchetti A, Galvan P, Sanchez S, Sanchez C, Hernandez $A$, et al. Relative dominance of Gag p24specific cytotoxic $\mathrm{T}$ lymphocytes is associated with human immunodeficiency virus control. / Virol 2006; 80:31223125.

40. Kelleher AD, Long C, Holmes EC, Allen RL, Wilson J, Conlon $\mathrm{C}$, et al. Clustered mutations in HIV-1 gag are consistently required for escape from HLA-B27-restricted cytotoxic T lymphocyte responses. J Exp Med 2001; 193: $375-386$.
41. Hollingsworth T, Anderson R, Fraser C. HIV-1 transmission, by stage of infection. J Infect Dis 2008; 198:687-693.

42. Brumme ZL, Goodrich J, Mayer HB, Brumme CJ, Henrick BM, Wynhoven B, et al. Molecular and clinical epidemiology of CXCR4-using HIV-1 in a large population of antiretroviralnaive individuals. I Infect Dis 2005; 192:466-474.

43. Herbeck JT, Müller V, Maust BS, Ledergerber B, Torti C, Di Giambenedetto $S$, et al. Is the virulence of HIV changing? A meta-analysis of trends in prognostic markers of HIV disease progression and transmission. AIDS 2012; 26:193-205. 